OPEN ACCESS

Edited by:

Artur Mayerhofer,

Ludwig Maximilian University

of Munich, Germany

Reviewed by:

Jens Vanselow,

Leibniz Institute for Farm Animal

Biology, Germany

Winnie Shum,

ShanghaiTech University, China

*Correspondence:

Jiwen Wang

wjw2886166@163.com

tThese authors have contributed

equally to this work

Specialty section:

This article was submitted to Reproduction

a section of the journal

Frontiers in Physiology

Received: 20 March 2020

Accepted: 14 May 2020

Published: 26 June 2020

Citation:

Chen X, Huang K, Hu S, Lan G,

Gan X, Gao S, Deng Y, Hu J, Li L, Hu B, He H, Liu H, Xia L and Wang J

(2020) FASN-Mediated Lipid

Metabolism Regulates Goose

Granulosa Cells Apoptosis and

Steroidogenesis.

Front. Physiol. 11:600.

doi: 10.3389/fphys.2020.00600

\section{FASN-Mediated Lipid Metabolism Regulates Goose Granulosa Cells Apoptosis and Steroidogenesis}

\author{
Xi Chen ${ }^{1+}$, Kailiang Huang ${ }^{2 t}$, Shenqiang Hu't, Gang Lan', Xiang Gan', Shanyan Gao', \\ Yan Deng', Jiwei Hu' ${ }^{1}$, Liang $\mathrm{Li}^{1}$, Bo Hu${ }^{1}$, Hua He ${ }^{1}$, Hehe Liu', Lu Xia ${ }^{1}$ and Jiwen Wang ${ }^{1 *}$ \\ ${ }^{1}$ Farm Animal Genetic Resources Exploration and Innovation Key Laboratory of Sichuan Province, College of Animal Science \\ and Technology, Sichuan Agricultural University, Chengdu, China, ${ }^{2}$ Key Laboratory of Agricultural Information Engineering \\ of Sichuan Province, College of Information Engineering, Sichuan Agricultural University, Ya'an, China
}

Lipid metabolism participates in regulating the functions of granulosa cells (GCs), which is important for follicular development. In this experiment, goose GCs from prehierarchical follicles and hierarchical follicles were selected to be the model for studying the putative regulatory role of lipid metabolism in apoptosis and steroidogenesis, through overexpression and interference with fatty acid synthase (FASN). When FASN was overexpressed, the lipid accumulation was increased in hierarchical GCs (hGCs) and it was increased in the two categorized GCs when FASN was interfered. In addition, the apoptosis of the two categorized GCs was increased when FASN was overexpressed, and their progesterone production was decreased when FASN was interfered. The results of qRT-PCR showed that, when FASN was overexpressed, the expression level of CYP11A1 was decreased in pre-hierarchical GCs (phGCs), while the expression levels of SCD1, DGAT2, APOB, and StAR were increased in hGCs. When FASN was interfered, the expression levels of CPT-1, DGAT2, and StAR were decreased whereas the expression level of CYP11A1 was increased in phGCs, and the expression levels of CPT-1, SCD1, and StAR were decreased in hGCs. These results not only identify the different effects of manipulated FASN expression on lipid metabolism of goose phGCs and hGCs but also demonstrate that FASN-mediated lipid metabolism plays an important role in regulating apoptosis and steroidogenesis of in vitro cultured goose GCs.

Keywords: lipid metabolism, granulosa cells, FASN, apoptosis, progesterone

\section{INTRODUCTION}

Female fertility depends on the normal development of ovarian follicles. As the key follicular component, granulosa cells (GCs) are essential for follicular growth, maturation, and ovulation due to their pivotal roles in regulating cellular apoptosis and steroidogenesis. In recent years, increased attention has been paid to lipid metabolism in GCs, and there are evidences that lipid metabolism in GCs is essential for maintaining ovarian follicle development in humans (Hu and Qiao, 2011) and other mammals (Campbell et al., 2010; Elis et al., 2015). Nevertheless, very little is known about the underlying mechanisms by which lipid metabolism in GCs modulates vertebrate ovarian functions. In contrast to the situation in mammals, lipid metabolism in GCs of avian may be 
more crucial and complicated because of the deposition of large amounts of liver-synthesized yolk precursors (mainly lipids) as the follicles develop. Besides, several lines of evidence showed that de novo lipogenesis (DNL) exists in the avian ovary including GCs, which may play important roles in regulating ovarian activities (Alvarenga et al., 2011). In support of this, our previously published study has demonstrated that fatty acid synthase (FASN), a key player in DNL, exhibited a stagedependent expression pattern in GCs from goose different-sized follicles (Wen et al., 2019). Thus, we hypothesized that FASNmediated lipid metabolism could be involved in regulating follicle development. More importantly, lipid metabolism dysfunction in GCs has been reported to be related to several ovarian diseases, such as GC tumor (Leung et al., 2019). Therefore, it is of great value to fully clarify the role of FASN-mediated lipid metabolism in GCs.

Lipids are not only the energy source and cell membrane components but also signal molecules that participated in the regulation of gene expression. Appropriate amount and composition of lipids are beneficial to cells; otherwise, cell dysfunction can be induced. Lipid metabolism has been associated with intracellular cholesterol concentration, since the inhibition of FASN by C75 (an inhibitor of FASN) significantly reduced cholesterol biosynthesis in cultured human macrophages (Rae and Graham, 2008). In vivo, cholesterol for steroidogenesis comes from the hydrolysis of cholesteryl ester in plasma lipoproteins, whereas in vitro, GCs may contribute to the supply of cholesterol since inhibition of cholesterol biosynthesis by simvastatin inhibited progesterone $\left(\mathrm{P}_{4}\right)$ biosynthesis in cultured bovine GCs (Spicer et al., 1996). A previous study in bovine GCs has suggested that inhibiting FASN by $\mathrm{C} 75$ reduced $\mathrm{P}_{4}$ biosynthesis (Elis et al., 2015). In addition, several studies have also correlated the process of lipid metabolism with cellular apoptosis in GCs. In bovine GCs, it was observed that excessive non-esterified fatty acids (NEFAs), especially saturated fatty acids (SFAs), could induce apoptosis (Vanholder et al., 2005), and it was also demonstrated in human GCs that SFAs have a positive effect on the apoptotic events (Mu et al., 2001). Significantly, downregulation of FASN also induced apoptosis in bovine GCs (Elis et al., 2015). Despite above efforts, the correlation of lipid metabolism with GC functions including steroidogenesis and apoptosis still seems to be far more intricate and remains largely unknown; further investigations are hence required.

In the present study, goose (Anser cygnoides) GCs were used as the research model to initially investigate the effects of manipulated FASN expression via the construction of the eukaryotic expression vector and RNA interference on lipid metabolism using laser scanning confocal microscopy (LSCM). Then, the effects of FASN-mediated lipid metabolism on GC steroidogenesis and apoptosis were determined using the ELISA and flow cytometry (FCM) methods, respectively. Finally, the differences and similarities of these effects were compared between goose ovarian prehierarchical GCs (phGCs) and hierarchical GCs (hGCs). These data are expected to provide new insights into the role of FASN-mediated lipid metabolism in regulating GC steroidogenesis and apoptosis.

\section{MATERIALS AND METHODS}

\section{Experimental Animals}

The maternal line of Tianfu meat geese, 35-40 weeks of age and laying in regular sequences of at least two to three eggs, was selected for this study. These geese were kept under the same conditions of light and temperature and were allowed ad libitum to feed and water at the Waterfowl Breeding Experimental Farm at Sichuan Agricultural University (Sichuan, China). Individual laying cycles were monitored and recorded, and the healthy geese were euthanized by cervical dislocation 6-8 $\mathrm{h}$ ahead of oviposition.

\section{Preparation of Recombinant Plasmid pEGFP-N1-FASN and FASN-Targeted SiRNA}

Specific primers for recombinant plasmid $p E G F P-N 1-F A S N$ construction and siRNA-FASN were designed according to the sequence of FASN (XM_013197939.1) available in GenBank. Three individual target fragments of FASN were amplified under the guidelines of PrimeSTAR Max Premix (TaKaRa, Dalian, China) and then ligated into the pEGFP$N 1$ vector previously linearized by BglII (NEB, Schwalbach, Germany) and HindIII (NEB, Schwalbach, Germany) with the In-Fusion@ HD Cloning Kit (Clontech, Mountain View, CA, United States) according to the manufacturer's instructions. Finally, the recombinant plasmid $p E G F P-N 1$ FASN was confirmed by restricted enzyme digestion, PCR, and sequencing. Detailed information on the primers of recombinant plasmid construction is summarized in Table 1 . siRNA-FASN-1 (5' GCUGGAUGCCAAUAGCUUUTT $\left.3^{\prime}\right)$ and siRNA-FASN-2 (5' GCUCGAUACCUUCCUGAAUTT $3^{\prime}$ ) were selected from three individual siRNAs due to their most efficiency in reducing FASN mRNA expression of phGCs and hGCs separately. In addition, a non-targeting (scrambled) siRNA was used as a control. Moreover, all siRNAs were designed and synthesized by Shanghai GenePharma Company (China).

\section{GCs Culture and Transfection}

The granulosa layers separating from pre-hierarchical (6$10 \mathrm{~mm}$ ) and hierarchical follicles (F4-F1) were washed with PBS (Solarbio, Beijing, China), respectively. GCs were isolated by $0.1 \%$ collagenase II digestion, then counted and further cultured into a 12-well culture plate or glass bottom cell culture dish, as previously described (Deng et al., 2018). At 70-80\% confluence, GCs were transfected with overexpression plasmid $(2 \mu \mathrm{g} /$ well of a 12 -well plate) and siRNA (90 pmol/well of a 12 -well plate), respectively, using Lipofectamine 3000 (Invitrogen, Carlsbad, CA, United States) according to the manufacturer's instructions and were assayed at $48 \mathrm{~h}$ post-transfection. 
TABLE 1 | Specific primers for amplification of FASN and construction of pEGFP-N1-FASN.

\begin{tabular}{|c|c|c|c|c|}
\hline Genes & Accession number & Primers $\left(5^{\prime}-3^{\prime}\right)$ & $\operatorname{Tm}\left({ }^{\circ} \mathrm{C}\right)$ & Size (bp) \\
\hline FASN-1 & XM_013197939.1 & F:agcgctaccggactcagatctATGGAGGACGTGGTGATTGCA R:TCCTAGCCAGATCACTITGCCA & 60 & 2197 \\
\hline FASN-2 & XM_013197939.1 & F:GCAAAGTGATCTGGCTAGGAATTC R:GTGTAGAAGTGCTGAAGTGGGGAA & 60 & 2516 \\
\hline FASN-3 & XM_013197939.1 & F:CCACTTCAGCACTTCTACACAACTAA R:cgactgcagaattcgaagcttTTAACСCTCTCTGACACTGACACGT & 60 & 2897 \\
\hline
\end{tabular}

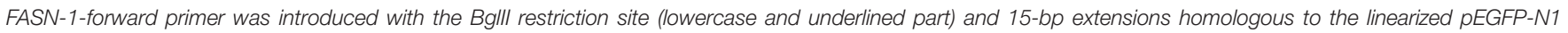

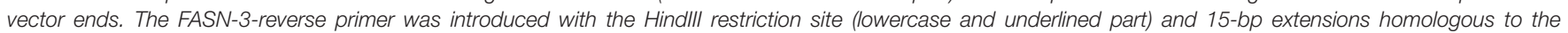

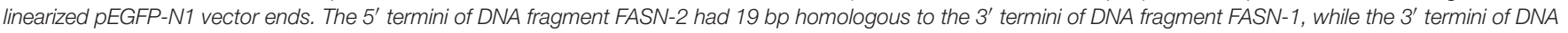
fragment FASN-2 had 19 bp homologous to the 5' termini of DNA fragment FASN-3.

\section{BODIPY 493/503 Staining of Lipid Droplets (LDs) for Morphological Observation by LSCM and Quantification by FCM}

The GCs growing on the glass bottom cell culture dish were fixed with $4 \%$ paraformaldehyde at room temperature for $30 \mathrm{~min}$ and then incubated in BODIPY (Thermo Fisher, Carlsbad, CA, United States) staining solution ( $1 \mu \mathrm{g} / \mathrm{ml}$, diluted in PBS) in the dark at $25^{\circ} \mathrm{C}$ for $15 \mathrm{~min}$ to label the LDs. Subsequently, GCs were washed three times with PBS and then the nuclei were labeled with Hoechst staining solution $(2 \mu \mathrm{g} / \mathrm{ml}$, diluted in PBS) for $15 \mathrm{~min}$. After that, GCs were washed for the final three times with PBS and incubated in $1 \mathrm{ml}$ PBS. Subsequently, the morphological characteristics of LDs were observed with a FV1200 LSCM (Olympus, Japan).

In addition, cell suspension was prepared by digestion with 0.25\% trypsin-EDTA (Gibco, Carlsbad, CA, United States), then washed one time with PBS. The cells were further incubated in BODIPY staining solution $(100 \mathrm{ng} / \mathrm{ml}$, diluted in PBS) in the dark at $25^{\circ} \mathrm{C}$ for $15 \mathrm{~min}$ to label the LDs, then washed three times with PBS, and resuspended with $200 \mu \mathrm{l}$ PBS. In the immediate aftermath of filtering, the mean fluorescence intensity (MFI) was measured using the BD Accuri C6 flow cytometer (Becton Dickinson, Franklin Lakes, NJ, United States) and analyzed using FlowJo software (Tree Star Inc., Ashland, OR, United States); 20,000 cells were analyzed per sample.

\section{Annexin V-FITC/PI Double Staining in the Detection of Apoptosis by FCM}

Cell suspension was prepared by digestion with $0.25 \%$ trypsin-EDTA, and then staining was performed with the Annexin V/PI cell apoptosis detection kit (Beyotime Biotech, Nantong, China) according to the manufacturer's instructions. Apoptotic cells were quantified using the BD Accuri C6 Flow cytometer and analyzed using FlowJo software; 20,000 cells were analyzed per sample, and the apoptotic rate was presented as the sum of early and late apoptosis subpopulations.

\section{ELISA for Determination of $\mathbf{P}_{\mathbf{4}}$ Production in the Supernatant Culture Medium}

The production of $\mathrm{P}_{4}$ in the supernatant culture medium was detected by goose $\mathrm{P}_{4}$ (PROG) ELISA Kit (Huding Biotechnology,
Shanghai, China) according to the manufacturer's instructions, and finally the $\mathrm{P}_{4}$ production of each well was normalized by the total RNA amount of the same well. The data were expressed as a percentage of $\mathrm{P}_{4}$ production by control.

\section{RNA Extraction and qRT-PCR Analysis}

The total RNA was extracted from each sample using TRIzol reagent (Invitrogen, Carlsbad, CA, United States) according to the manufacturer's instructions, then the RNA quality, purity, and concentration were measured by spectrophotometric absorbance measurement. The cDNA was further synthesized from $1 \mu \mathrm{g}$ of total RNA using a PrimeScript $\mathrm{RT}^{\mathrm{TM}}$ Reagent Kit (TaKaRa, Dalian, China), in accordance with the manufacturer's instructions. The qRT-PCR analysis was conducted using $2 \times$ SYBR Premix Ex Taq II (TaKaRa, Dalian, China). The reaction solution was prepared in a total volume of $12.5 \mu \mathrm{l}$ containing $1 \mu \mathrm{l}$ cDNA, $6.25 \mu \mathrm{l}$ of $2 \times$ SYBR Premix Ex Taq, $4.25 \mu \mathrm{l}$ of $\mathrm{ddH}_{2} \mathrm{O}$, and $0.5 \mu \mathrm{l}$ of each genespecific primer $(10 \mu \mathrm{M})$. For each sample, the analysis was conducted in triplicate and normalized to $\beta$-actin by the $2^{-\Delta}$ $\Delta \mathrm{Ct}$ method (Livak and Schmittgen, 2000). The control was set as one. The primers for qRT-PCR are summarized in Table 2.

\section{Statistical Analysis}

All experimental data were subjected to statistical analysis by Student's $t$-test. All statistical analyses were performed using SPSS software (SPSS Inc., Chicago, IL, United States). Results were presented as the mean \pm SEM of three independent experiments. $P<0.05$ was considered as significant.

\section{RESULTS}

\section{Efficiency of FASN Overexpression and Interference}

Three individual target fragments of FASN were obtained by PCR amplification. Then, the recombinant plasmid $p E G F P-N 1$ FASN was constructed and confirmed by BglII and HindIII double digestion. The expression level of FASN after the treatment with $F A S N$ overexpression and interference was further determined by qRT-PCR. For the overexpression group, the expression levels of FASN increased to almost 19- and 76fold compared to the control (untransfected cells) in phGCs and hGCs, respectively (Figure 1A). In addition, it was worth mentioning that the empty $p E G F P-N 1$ vector had no influence 
TABLE 2 | Primers for qRT-PCR.

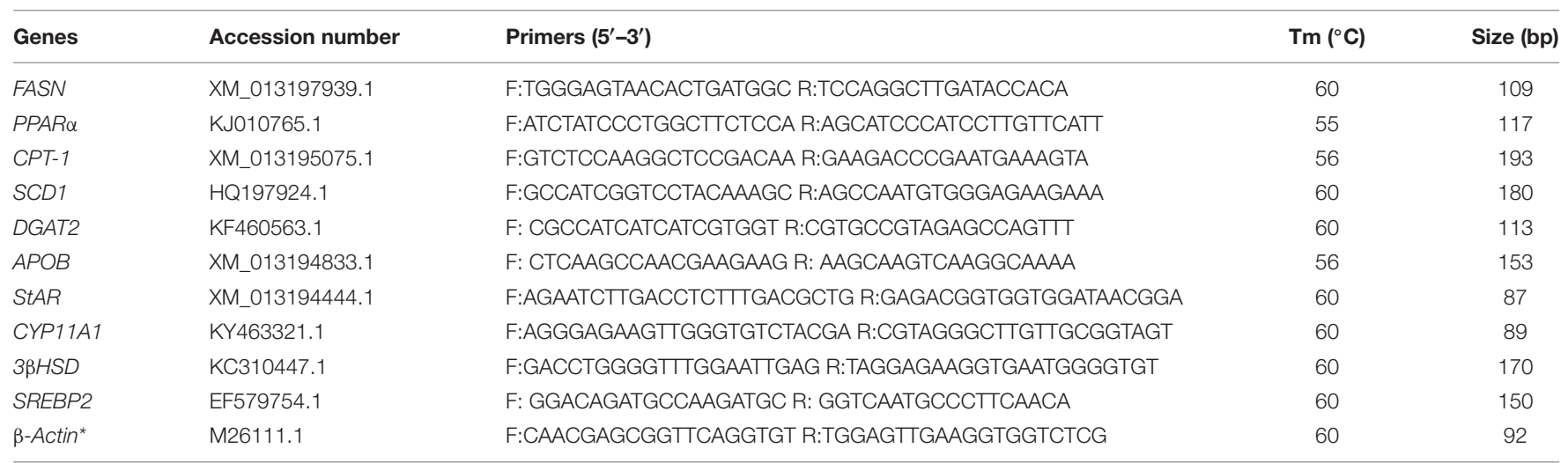

F: sense primers; R: antisense primers. *House-keeping gene for data normalization.
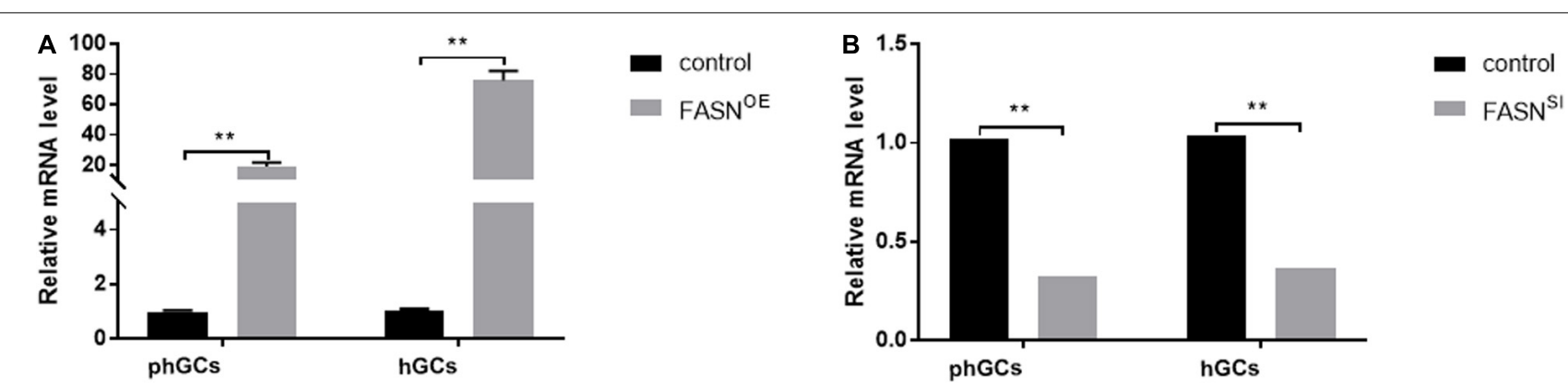

FIGURE 1 | Efficiency of FASN overexpression or interference. (A) The expression levels of FASN of phGCs and hGCs were detected by qRT-PCR 48 h after treatment with FASN overexpression, respectively. (B) The expression levels of FASN of phGCs and hGCs were detected by qRT-PCR $48 \mathrm{~h}$ after treatment with FASN interference, respectively. OE, overexpression; SI, RNA interference; phGCs, pre-hierarchical GCs; hGCs, hierarchical GCs. The expression of FASN was normalized by $\beta$-actin, and the control was set as one. Results were presented as the mean \pm SEM of three independent experiments (** $P<0.01$ ).

on the cell viability and FASN expression level of phGCs and hGCs (Supplementary Figure S1). For the interference group, the expression levels of FASN reduced to 0.33- and 0.37-fold compared to the control in phGCs and hGCs, respectively (Figure 1B).

\section{Effects of Overexpressing and Interfering FASN on Lipid Accumulation in phGCs and hGCs}

The morphological characteristics (Figure 2A) and quantification of LDs (Figure 2B) were measured by LSCM and FCM, respectively. When FASN was overexpressed, compared to the control, the content of LDs increased significantly in hGCs $(P<0.01)$ whereas no significant change was observed in phGCs $(P>0.05)$. When FASN was interfered, compared to the control, the content of LDs increased significantly in both phGCs and hGCs $(P<0.01)$.

\section{Effects of Overexpressing and Interfering FASN on Apoptosis of phGCs and hGCs}

The apoptosis was determined by FCM, and the results are displayed in Figures 3A,B. Overexpressing FASN significantly increased the apoptotic rate of both phGCs $(P<0.01)$ and hGCs
$(P<0.05)$; however, interfering FASN showed no significant effect on apoptosis in either phGCs or hGCs $(P>0.05)$.

\section{Effects of Overexpressing and Interfering FASN on $\mathrm{P}_{4}$ Production of phGCs and hGCs}

The production of $\mathrm{P}_{4}$ in the supernatant culture medium was determined by the ELISA method, and the results are displayed in Figure 4. Overexpressing FASN had no significant effect on $\mathrm{P}_{4}$ production in both phGCs $(P>0.05)$ (Figure 4A) and hGCs $(P>0.05)$ (Figure 4B), while interfering FASN significantly reduced the $\mathrm{P}_{4}$ production in both phGCs $(P<0.01)$ (Figure 4C) and hGCs $(P<0.05)$ (Figure 4D).

\section{Effects of Overexpressing and Interfering FASN on the Expression Levels of Genes Involved in Lipid Metabolism and Steroidogenesis}

The expression levels of lipid metabolism- and steroidogenesisrelated genes were assessed by qRT-PCR, and the results are displayed in Figure 5. When FASN was overexpressed, in phGCs (Figure 5A), the expression levels of perixisome proliferationactivated receptor alpha $(P P A R \alpha)$, carnitine palmitoyltransferase 

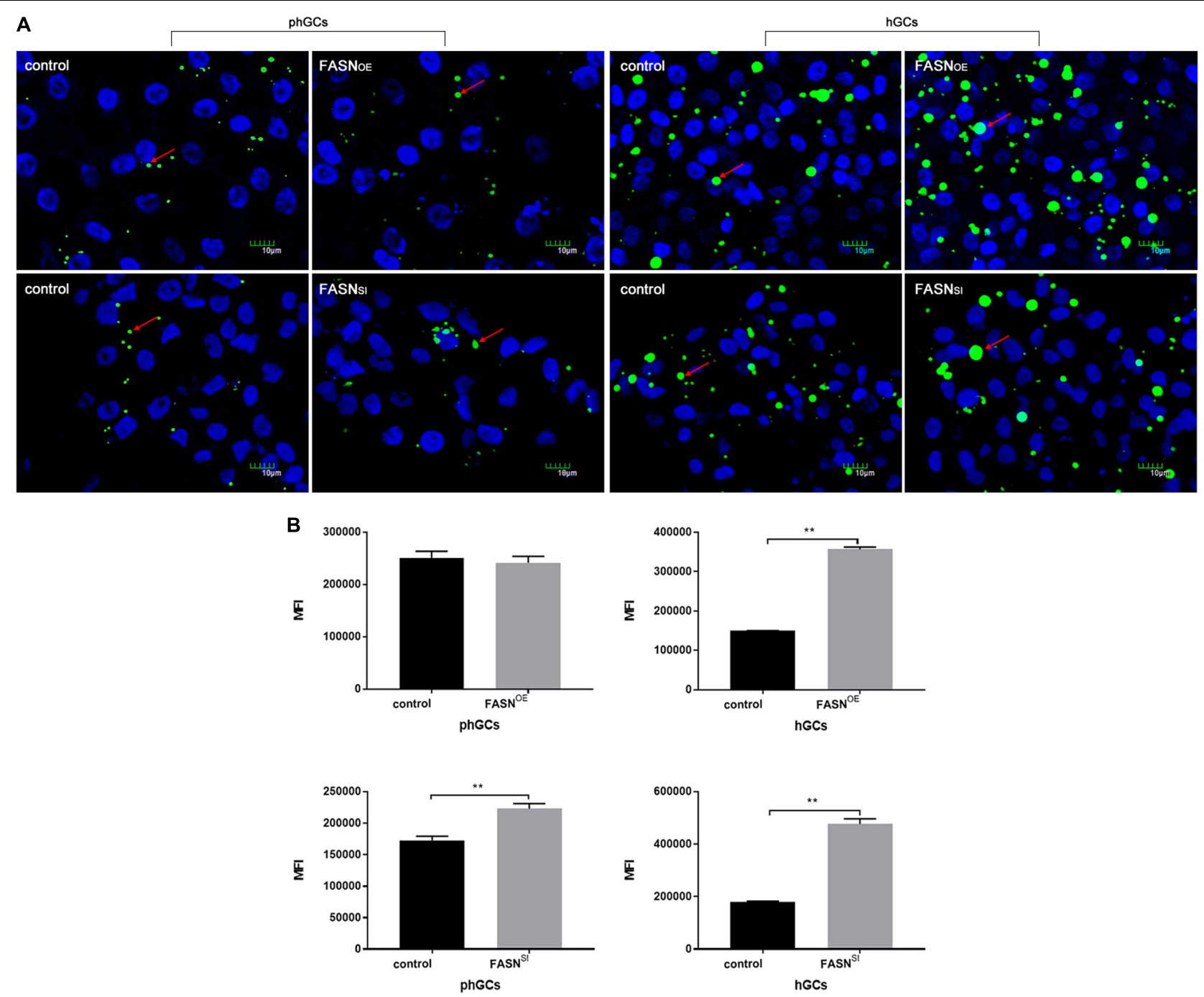

FIGURE 2 | Effects of FASN overexpression or interference on lipid accumulation in cultured phGCs and hGCs. (A) Morphological characteristics of phGCs and hGCs were observed by LSCM $48 \mathrm{~h}$ after treatment with FASN overexpression and interference, respectively. The red arrows represent LDs. The scale marker represents $10 \mu \mathrm{m}$. (B) MFI of phGCs and hGCs was determined by FCM $48 \mathrm{~h}$ after treatment with FASN overexpression and interference, respectively. 20,000?cells per sample were considered in the gated regions for calculations. OE, overexpression; SI, RNA interference; phGCs, pre-hierarchical GCs; hGCs, hierarchical GCs; MFI, mean fluorescence intensity; FCM, flow cytometry; LSCM, laser scanning confocal microscopy. Results were presented as the mean \pm SEM of three independent experiments ( $\left.{ }^{\star \star} P<0.01\right)$.

1 (CPT-1), stearoyl-CoA desaturase 1 (SCD1), diacylglycerol acyltransferase 2 (DGAT2), apolipoprotein B (APOB), steroidogenic acute regulatory $(S t A R)$ protein, $3 \beta$-hydroxysteroid dehydrogenase $(3 \beta H S D)$, and sterol regulatory element binding protein-2 (SREBP2) were not significantly affected $(P>0.05)$, whereas the expression level of recombinant cytochrome $\mathrm{P} 450$ 11A1 (CYP11A1) was reduced significantly $(P<0.05)$; in hGCs (Figure 5B), the expression levels of SCD1 $(P<0.01)$, DGAT2 $(P<0.01), A P O B(P<0.05)$, and $\operatorname{StAR}(P<0.05)$ were increased significantly, while the expression levels of $P P A R \alpha, C P T-1$, CYP11A1, 3BHSD, and SREBP2 were not significantly affected $(P>0.05)$. When FASN was interfered, in phGCs (Figure 5C), the expression levels of $C P T-1(P<0.01)$, DGAT2 $(P<0.05)$, and $\operatorname{StAR}(P<0.01)$ were decreased significantly, whereas the expression levels of CYP11A1 were increased significantly $(P<0.01)$, and no significant effects were seen in the expression levels of PPAR $\alpha, S C D 1, A P O B, 3 \beta H S D$, and SREBP2 $(P>0.05)$; in hGCs (Figure 5D), the expression levels of CPT-1, SCD1, and StAR were reduced significantly $(P<0.01)$, while the expression levels of PPAR $\alpha, D G A T 2, A P O B, C Y P 11 A 1$, and $3 \beta H S D$ and $S R E B P 2$ were not significantly affected $(P>0.05)$.

\section{DISCUSSION}

To our knowledge, this study represents the first to determine the effects of FASN on lipid metabolism of phGCs and hGCs and to explore the influence of FASN-mediated lipid metabolism 

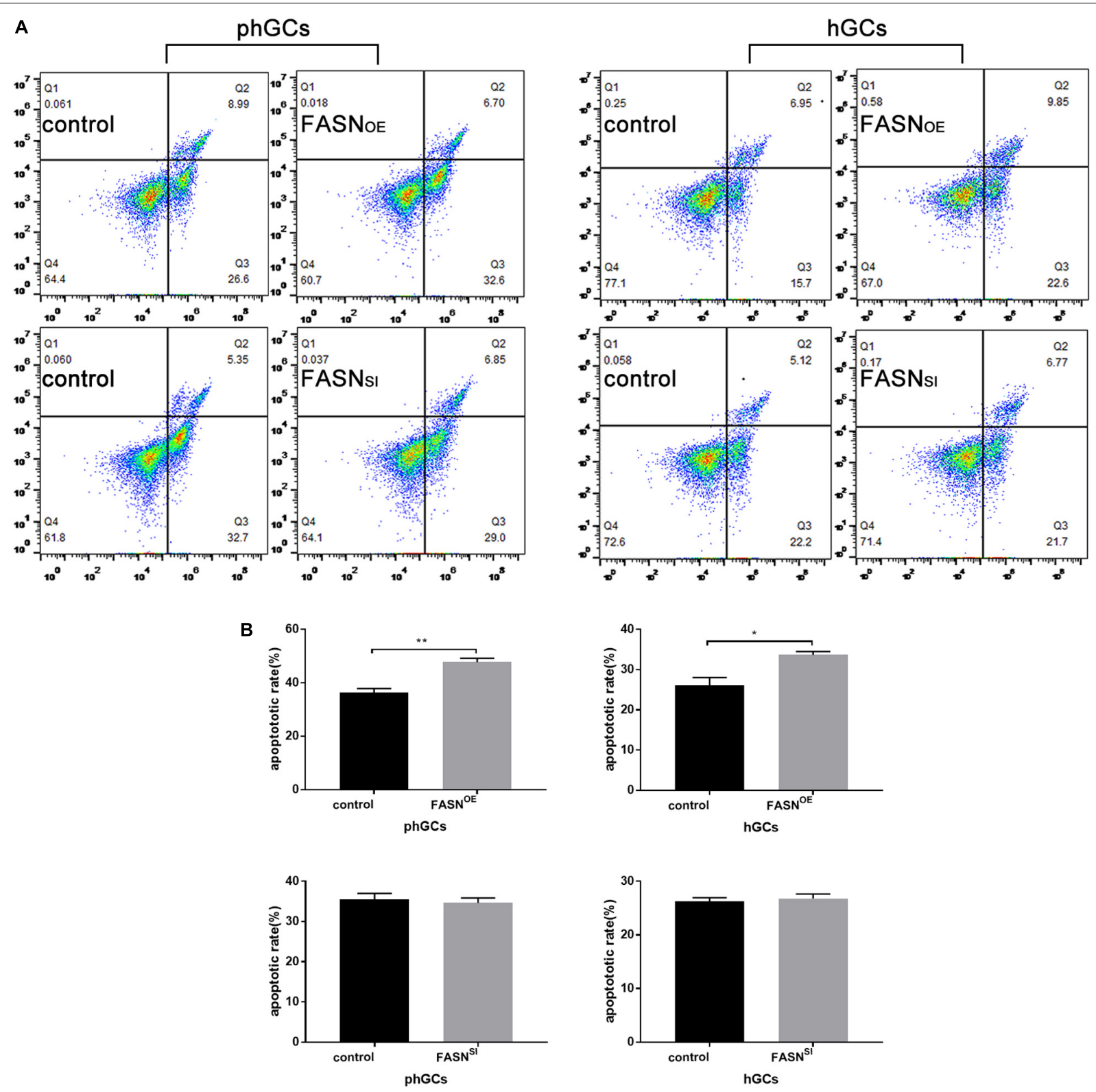

FIGURE 3 | Effects of FASN overexpression or interference on apoptosis of cultured phGCs and hGCs. (A) Apoptosis of phGCs and hGCs was determined by FCM $48 \mathrm{~h}$ after treatment with FASN overexpression and interference, respectively. The upper-left (Q1), upper-right (Q2), lower-right (Q3), and lower-left (Q4) quadrants in each panel represent the populations of cell debris, late apoptotic and necrotic, early apoptotic, and normal cells, respectively. (B) The overall apoptotic rate was the sum of early and late apoptosis subpopulations. 20,000?cells per sample were considered in the gated regions for calculations. OE, overexpression; SI, RNA interference; phGCs, pre-hierarchical GCs; hGCs, hierarchical GCs; FCM, flow cytometry. Results were presented as the mean \pm SEM of three independent experiments $\left({ }^{\star} P<0.05,{ }^{\star \star} P<0.01\right)$.

on GC steroidogenesis and apoptosis in geese. Previous studies have indicated that upregulation of FASN induced increased lipid accumulation in bovine (Chu et al., 2018b) and murine (Chu et al., 2018a) mammary epithelial cells. Consistent with this, our data also showed that overexpressing FASN increased the amount of LDs in goose hGCs although it appeared to have no effect on those in phGCs. Furthermore, overexpressing FASN increased the mRNA levels of SCD1 and DGAT2 (two key regulatory enzymes in DNL) in hGCs instead of phGCs.
It has been reported that once palmitic acid is obtained, it can be de-saturated by SCD1 (Strable and Ntambi, 2010). These unsaturated fatty acids (USFAs) can be further esterified into triglyceride (TG) through the catalysis of DGAT2 and then be stored into LDs (Ameer et al., 2014). Thus, it would be understandable that overexpression of FASN failed to promote lipid accumulation in phGCs because it did not induce similar changes in expression of DNL-related genes in phGCs to those in hGCs. From Figure 5B, overexpressing 

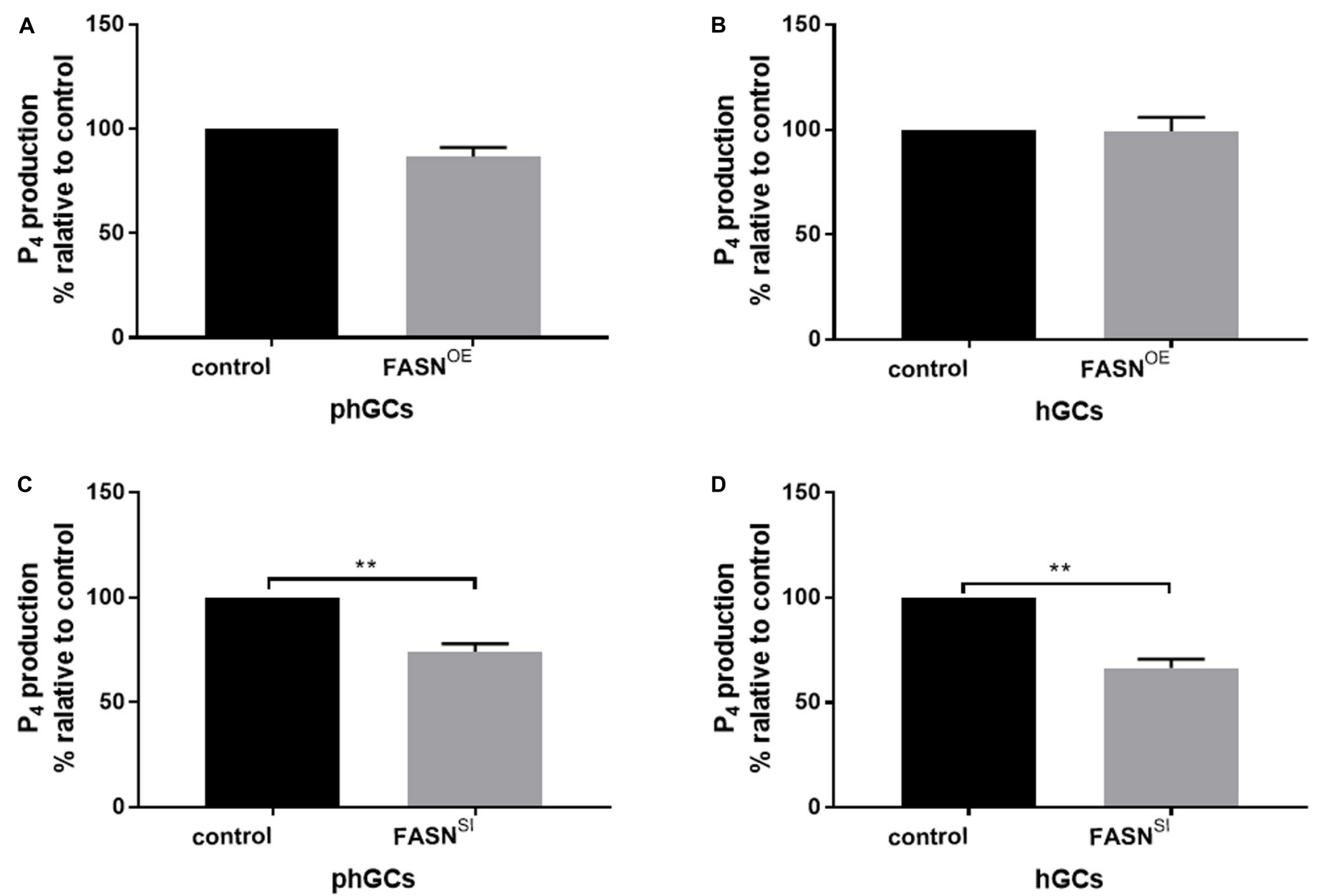

FIGURE 4 | Effects of FASN overexpression or interference on $\mathrm{P}_{4}$ production of cultured phGCs and hGCs. $\mathrm{P}_{4}$ production of phGCs (A) and hGCs (B) was determined by the ELISA method $48 \mathrm{~h}$ after treatment with FASN overexpression, respectively. $\mathrm{P}_{4}$ production of phGCs (C) and hGCs (D) was determined by the ELISA method $48 \mathrm{~h}$ after treatment with FASN interference as determined by the ELISA method, respectively. OE, overexpression; SI, RNA interference; phGCs, pre-hierarchical GCs; hGCs, hierarchical GCs; $P_{4}$, progesterone. $P_{4}$ production was normalized by the total RNA amount of the well and expressed as a percentage of $\mathrm{P}_{4}$ production by control. Results were presented as the mean $\pm \mathrm{SEM}$ of three independent experiments $\left.{ }^{\star \star} P<0.01\right)$.

FASN also increased the mRNA levels of $A P O B$ (a gene participated in the regulation of lipoprotein assembly and secret) in hGCs, from which we inferred that the dramatically increased deposition might promote the transport of lipids from intracellular to the extracellular, which would affect the content of yolk lipids.

In addition, a previous study showed that downregulation of CPT-1 (a key regulatory enzyme in the $\beta$-oxidation of longchain fatty acids) induced an increase in intracellular lipid content of porcine adipocyte (Zhang et al., 2014). Our results showed that in both phGCs and hGCs, the significantly increased amount of LDs was accompanied by the decrease in CPT1 when FASN was interfered and which was similar to the previous report. Free fatty acids (FFAs) are the endogenous ligand of $P P A R \alpha$, and the receptor ligand complexes can further activate CPT-1 expression (Huang et al., 2012). After prolonged fasting, FASKOL (FASN knockout in liver) mice developed fatty liver, similarly to fasted $P P A R \alpha$-deficiency mice (Chakravarthy et al., 2005). However, the interference of FASN did not affect the expression of $P P A R \alpha$ in this study. It indicated that the reduction in PPAR $\alpha$ ligand instead of PPAR $\alpha$ caused by FASN downregulation induced an inhibition of the $P P A R \alpha-C P T-1$ pathway, which should be responsible for the marked increase in lipid accumulation after downregulating FASN in our study. Moreover, the expression of DGAT2 in the two categorized GCs showed a decreasing trend although the decrease was not significant in hGCs. Thus, we speculated that the increasing content of LDs was irrelevant to the genes related to DNL when FASN was interfered. Taken together, the mechanism of regulating lipid metabolism by FASN in phGCs was inconsistent with that in hGCs. Specifically, FASN could participate in regulating the lipid content in phGCs mostly by activating the $P P A R \alpha-C P T-1$ pathway but could regulate it in hGCs by activating both the DNL (FASN$S C D 1 / D G A T 2 / A P O B)$ pathway and the lipid oxidation $(P P A R \alpha-$ $C P T-1)$ pathway.

Saturated fatty acids could induce apoptosis not only in GCs but also in other cell types (Jorritsma et al., 2004; Vanholder et al., 2005, 2006). In this study, the apoptotic rate of both phGCs and hGCs significantly increased by overexpression of FASN rather than interference. Previous studies reported that the conversion of SFAs into USFAs by 


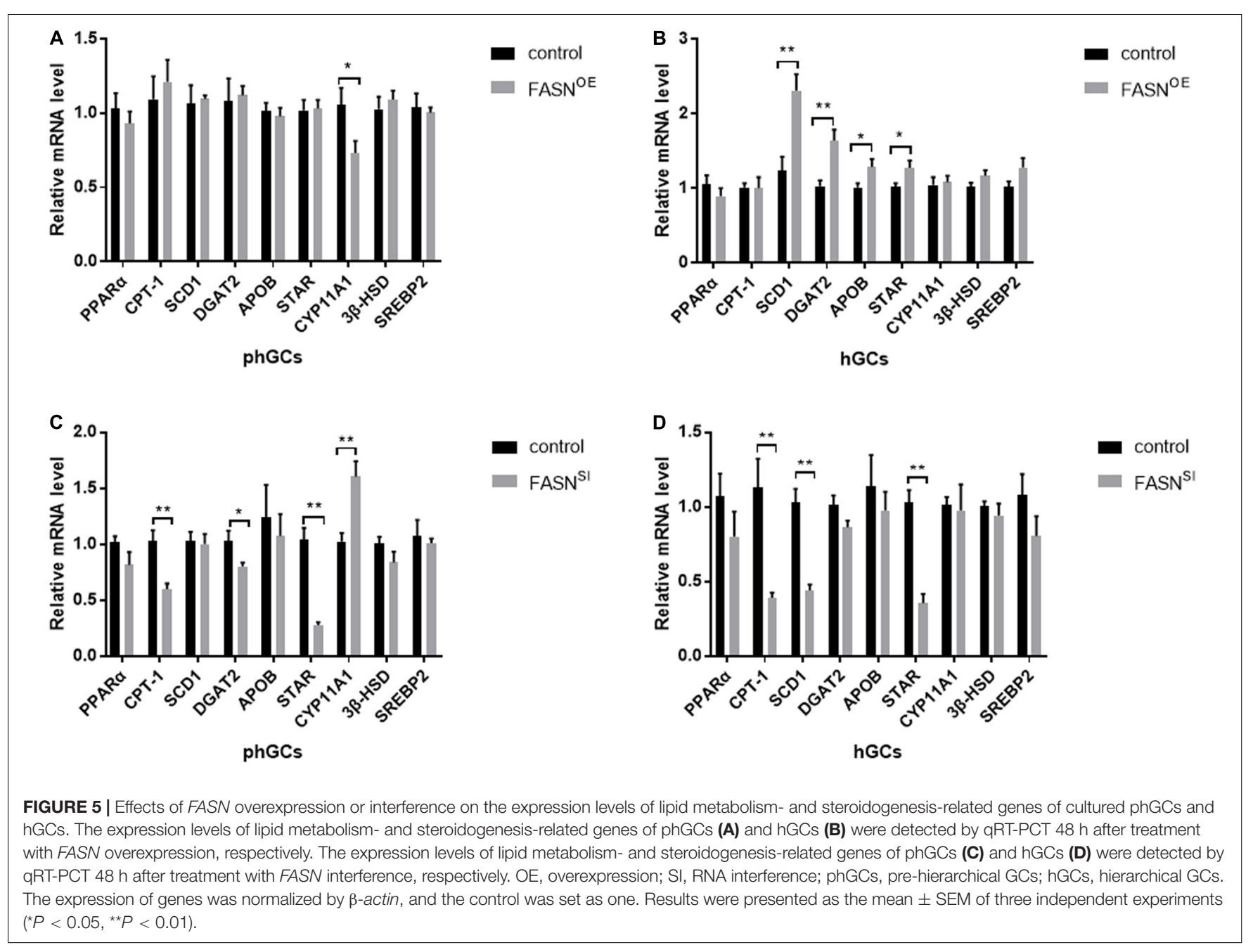

SCD1 in cumulus cells (a type of GC) protected cells against lipid-induced damage, such as apoptosis, through promoting the distribution of fatty acids toward LDs (Listenberger et al., 2003; Aardema et al., 2017). SCD1 provides a more accessible pool of USFAs for DGAT2 for TG synthesis through substrate channeling (Man et al., 2006). As discussed earlier, overexpressing FASN had no significant effects on the amount of LDs in phGCs, as well as the expression of SCD1 and DGAT2. This led to a conclusion that phGCs were negatively affected by the dramatically increased but not effectively de-saturated fatty acids caused by FASN overexpression, and which finally induced the increase of apoptosis. However, overexpressing FASN increased SCD1 and DGAT2 gene expression of hGCs to about 2-fold and 1.6-fold, respectively, and the interaction of these two enzymes could protect hGCs against lipid-induced damage. However, the apoptosis of hCGs still increased, though in a less intense form compared to that in phGCs. Considering that the expression of FASN increased 76-fold after overexpressing, the slightly increasing SCD1 and DGAT2 appeared to be inadequate to totally convert the massive SFAs to USFAs and then to TG. It indicated that hGCs were capable of preventing themselves from apoptosis induced by lipid metabolism dysfunction through activating
SCD1 and DGAT2 for finally channeling SFAs into TG pools and storing into LDs. FASN is preferentially overexpressed in cancer cells and has been strongly linked to cancer cell proliferation and migration (Jiang et al., 2012). Plenty of previous studies on cancer cells showed that inhibiting FASN can increase apoptosis (Gonzalezguerrico et al., 2008; Richa et al., 2015; Sun et al., 2018; You et al., 2019). However, the demands of phGCs and hGCs for FASN are obviously lower than those of cancer cells, so the sensitivity of GCs to FASN inhibition may also be lower than that of cancer cells. Taken together, our results suggested that although the apoptosis induced by FASN-mediated lipid metabolism in the two categorized GCs was similar, expressions of SCD1 and DGAT2 were positively correlated with those of FASN in hGCs but not in phGCs. This difference might indicate that, during early stages of follicle development, GCs could not protect themselves from apoptosis induced by lipid metabolism dysfunction through activating the expression of SCD1 and DGAT2. Moreover, such capacity would be given to GCs with the development of follicles.

Cholesterol in the cytoplasm can be delivered to the mitochondrial inner membrane by $S t A R$, then be converted to pregnenolone by $C Y P 11 A 1$, and these pregnenolones 
are later converted to $\mathrm{P}_{4}$ by $3 \beta H S D$ in mitochondria (Marion and $\mathrm{Li}, 2008$ ). In this study, $\mathrm{P}_{4}$ production and the expression of StAR in both phGCs and hGCs were decreased by interference of FASN rather than overexpression. It indicated that the inhibition of FASN reduced $\mathrm{P}_{4}$ production by preventing cholesterol from entering the mitochondrial inner membrane. In vivo, reduction in $\mathrm{P}_{4}$ has been reported to cause ovulation disorder in avian (Etches, 1979) and fail to protect luteal cells from apoptosis induced by $P R L$ (prolactin) in mammals (Okuda et al., 2004). Unlike the interference, the overexpression of FASN had no effect on $\mathrm{P}_{4}$ production for those two categorized GCs. As shown in Figure 5A, in phGCs, the unchanged expression levels of StAR, CYP11A1, and $3 \beta H S D$ might be the reason for the failure of $\mathrm{P}_{4}$ production to respond to the increasing fatty acid de novo synthesis. In hGCs, although the cholesterol entering the mitochondrial inner membrane probably increased with the increase in StAR expression, $\mathrm{P}_{4}$ production is still limited by the limited effectiveness of catalysis by CYP11A1 and $3 \beta H S D$, as shown in Figure 5B. Despite that the responses of the steroidogenesis-related gene expression levels in phGCs were not that consistent with those in hGCs for the stimulation of FASN overexpression, the result was no effect on $\mathrm{P}_{4}$ production in the two categorized GCs. Besides the stimulation of gonadotropins (Rivas et al., 2015), steroidogenesis is also stimulated by other physiological active substances existing in the follicles (Groothuis et al., 2005). According to our results, the increasing fatty acids induced by overexpression of FASN were not such substances that could effectively stimulate the steroidogenesis in goose GCs from the follicles at different stages of development. Additionally, we also measured the mRNA levels of SREBP2, which is a master regulator of intracellular cholesterol homeostasis. It has been reported that, when cells are deprived of cholesterol, SREPB2 would be activated then lead to enhanced cholesterol uptake and biosynthesis (Miserez et al., 2002). However, in this study, SREBP2 was not affected by FASN overexpression or interference either in phGCs or hGCs. Thus, it could be inferred that the decrease in $\mathrm{P}_{4}$ production in both phGCs and hGCs induced by FASN interference is probably not due to the decrease in intracellular cholesterol. However, it has been proved that, in bovine GCs, the reduction in $\mathrm{P}_{4}$ biosynthesis by inhibiting FASN was likely related to the potential inhibition of cholesterol biosynthesis (Elis et al., 2015). However, our results indicated that, in goose GCs, FASN participated in the regulation of steroidogenesis through StAR which can modulate the amount of cholesterol that enters the mitochondrial inner membrane. This demonstrated that the ways for FASN-mediated lipid metabolism to involve in regulating steroidogenesis of GCs could be various.

\section{CONCLUSION}

In summary, this study not only examined the effects of manipulated FASN expression on lipid metabolism of goose phGCs and hGCs but also explored the effects of FASN-mediated lipid metabolism on cellular apoptosis and steroidogenesis. The most interesting finding was that lipid metabolic homeostasis appeared to be one of the prerequisites for GCs to maintain its normal function. Specifically, FASN-mediated lipid metabolism can regulate GC apoptosis, which may be related to lipotoxicity; besides, our study provides new mechanistic insights into the regulation of $\mathrm{P}_{4}$ production by FASN-mediated lipid metabolism, in which the regulation of StAR by FASN is critical. These findings are expected to provide a theoretical support for further elucidating the mechanism of lipid metabolism in regulating the function of avian GCs throughout follicular development.

\section{DATA AVAILABILITY STATEMENT}

The raw data supporting the conclusions of this article will be made available by the authors, without undue reservation.

\section{ETHICS STATEMENT}

All procedures in this study were approved by the Faculty Animal Care and Use Committee of Sichuan Agricultural University (Sichuan, China).

\section{AUTHOR CONTRIBUTIONS}

$\mathrm{XC}$ contributed to methodology, software, validation, formal analysis, investigation, resources, writing-original draft preparation, writing-review and editing, and visualization. $\mathrm{KH}$ contributed to software and investigation. SH contributed to conceptualization, data curation, writing-review and editing, funding acquisition, and project administration. GL, XG, SG, $\mathrm{YD}, \mathrm{JH}, \mathrm{LL}, \mathrm{BH}, \mathrm{HH}, \mathrm{HL}$, and LX contributed to investigation. JW contributed to supervision, project administration, and funding acquisition.

\section{FUNDING}

This work was supported by the National Natural Science Foundation of China (31672424, 31802064, and 31972567), the Sichuan Science and Technology Program (2019YJ0417), the China Agricultural Research System (CARS-42-4), and the Livestock \& Poultry Breeding Research Project of Sichuan Province (No. 2016NYZ0044).

\section{ACKNOWLEDGMENTS}

The authors wish to thank Dong Li for his technical assistance.

\section{SUPPLEMENTARY MATERIAL}

The Supplementary Material for this article can be found online at: https://www.frontiersin.org/articles/10.3389/fphys.2020. 00600/full\#supplementary-material

FIGURE S1 | Effect of pEGFP-N1 vector on cell viability and FASN expression level of phGCs and hGCs. 


\section{REFERENCES}

Aardema, H., van Tol, H. T. A., Wubbolts, R. W., Brouwers, J. F. H. M., Gadella, B. M., and Roelen, B. A. J. (2017). Stearoyl-CoA desaturase activity in bovine cumulus cells protects the oocyte against saturated fatty acid stress. Biol. Reprod. 96, 982-992. doi: 10.1095/biolreprod.116.146159

Alvarenga, R. R., Zangeronimo, M. G., Pereira, L. J., Rodrigues, P. B., and Gomide, E. M. (2011). Lipoprotein metabolism in poultry. Worlds Poult. Sci. J. 67, 431-440. doi: 10.1017/s0043933911000481

Ameer, F., Scandiuzzi, L., Hasnain, S., Kalbacher, H., and Zaidi, N. (2014). De novo lipogenesis in health and disease. Metabolism 63, 895-902. doi: 10.1016/j. metabol.2014.04.003

Campbell, B. K., Onions, V., Kendall, N. R., Guo, L., and Scaramuzzi, R. J. (2010). The effect of monosaccharide sugars and pyruvate on the differentiation and metabolism of sheep granulosa cells in vitro. Reproduction 140, 541-550. doi: 10.1530/REP-10-0146

Chakravarthy, M. V., Pan, Z. J., Zhu, Y. M., Tordjman, K., Schneider, J. G., Coleman, T., et al. (2005). "New" hepatic fat activates PPAR $\alpha$ to maintain glucose, lipid, and cholesterol homeostasis. Cell Metabol. 1, 309-322. doi: 10. 1016/j.cmet.2005.04.002

Chu, M., Zhao, Y., Yu, S., Hao, Y., Zhang, P., Feng, Y., et al. (2018a). MicroRNA221 may be involved in lipid metabolism in mammary epithelial cells. Int. J. Biochem. Cell Biol. 97, 118-127. doi: 10.1016/j.biocel.2018.02.014

Chu, M., Zhao, Y., Yu, S., Hao, Y., Zhang, P., Feng, Y., et al. (2018b). miR-15b negatively correlates with lipid metabolism in mammary epithelial cells. Am. J. Physiol. Cell Physiol. 314, C43-C52. doi: 10.1152/ajpcell.00115.2017

Deng, Y., Gan, X., Chen, D., Huang, H. L., Yuan, J. S., Qiu, J. M., et al. (2018). Comparison of growth characteristics of in vitro cultured granulosa cells from geese follicles at different developmental stages. Biosci. Rep. 38, 1-12. doi: 10. 1042/BSR20171361

Elis, S., Desmarchais, A., Maillard, V., Uzbekova, S., Monget, P., and Dupont, J. (2015). Cell proliferation and progesterone synthesis depend on lipid metabolism in bovine granulosa cells. Theriogenology 83, 840-853. doi: 10.1016/ j.theriogenology.2014.11.019

Etches, R. J. (1979). Plasma concentrations of progesterone and corticosterone during the ovulation cycle of the Hen (Gallus Domesticus). Poul. Sci. 58, 211-216. doi: 10.3382/ps.0580211

Gonzalezguerrico, A., Espinoza, I., Dimri, M., Loda, M., and Lupu, R. (2008). Fatty Acid Synthase (FASN) promotes transformation of normal breast epithelial cells: a novel metabolic oncogene? Cancer Res. 68, 12-16.

Groothuis, T. G. G., Wendt, M., Nikolaus, V. E., Claudio, C., and Corine, E. (2005). Maternal hormones as a tool to adjust offspring phenotype in avian species. Neurosci. Biobehav. Rev. 29, 329-352. doi: 10.1016/j.neubiorev.2004.12.002

Hu, W. H., and Qiao, J. (2011). Expression and regulation of adipocyte fatty acid binding protein in granulosa cells and its relation with clinical characteristics of polycystic ovary syndrome. Endocrine 40, 196-202. doi: 10.1007/s12020-0119495-9

Huang, J. S., Jia, Y. Z., Fu, T., Viswakarma, N., Bai, L., Rao, M. S., et al. (2012). Sustained activation of PPAR $\alpha$ by endogenous ligands increases hepatic fatty acid oxidation and prevents obesity in ob/ob mice. FASEB J. 26, 628-638. doi: 10.1096/fj.11-194019

Jiang, B., Li, E. H., Lu, Y. L., Jiang, Q., Cui, D., Jing, Y. F., et al. (2012). Inhibition of fatty-acid synthase suppresses P-AKT and induces apoptosis in bladder cancer. Urology 80, .e9-.e484. doi: 10.1016/j.urology.2012.02.046

Jorritsma, R., César, M. L., Hermans, J. T., Kruitwagen, C. L. J. J., Vos, P. L. A. M., and Kruip, T. A. M. (2004). Effects of non-esterified fatty acids on bovine granulosa cells and developmental potential of oocytes in vitro. Anim. Reprod. Sci. 81, 225-235. doi: 10.1016/j.anireprosci.2003.10.005

Leung, D. T. H., Rainczuk, A., Nguyên, T., Stephens, A., Silke, J., Fuller, P. J., et al. (2019). Targeting XIAP and PPAR $\gamma$ in granulosa cell tumors alters metabolic signaling. J. Proteome Res. 18, 1691-1702. doi: 10.1021/acs.jproteome.8b00917

Listenberger, L. L., Han, X., Lewis, S. E., Cases, S., Farese, R. V., Ory, D. S., et al. (2003). Triglyceride accumulation protects against fatty acid-induced lipotoxicity. Proc. Natl. Acad. Sci. U.S.A. 100, 3077-3082. doi: 10.1073/pnas. 0630588100

Livak, K. J., and Schmittgen, T. D. (2000). Analysis of relative gene expression data using real-time quantitative PCR and the $2^{-\Delta \Delta \mathrm{Ct}}$ method. Methods 25, 402-408. doi: 10.1006/meth.2001
Man, W. C., Miyazaki, M., Chu, K., and Ntambi, J. (2006). Colocalization of SCD1 and DGAT2: implying preference for endogenous monounsaturated fatty acids in triglyceride synthesis. J. Lipid Res. 47, 1928-1939. doi: 10.1194/jlr.M600172JLR200

Marion, B. S., and Li, D. H. (2008). Regulation of steroid hormone biosynthesis by the cytoskeleton. Lipids 43, 1109-1115. doi: 10.1007/s11745-008-3221-2

Miserez, A. R., Muller, P. Y., Barella, L., Barella, S., Staehelin, H. B., Leitersdorf, E., et al. (2002). Sterol-regulatory element-binding protein (SREBP)-2 contributes to polygenic hypercholesterolaemia. Atherosclerosis 164, 15-26. doi: 10.1016/ s0021-9150(01)00762-6

Mu, Y. M., Yanase, T., Nishi, Y., Tanaka, A., Satio, M., Jin, C. H., et al. (2001). Saturated FFAs, palmitic acid and stearic acid, induce apoptosis in human granulosa cells. Endocrinology 142, 3590-3597. doi: 10.1210/endo.142.8.8293

Okuda, K., Korzekwa, A., Shibaya, M., Murakami, S., Nishimura, R., Tsubouchi, M., et al. (2004). Progesterone is a suppressor of apoptosis in bovine luteal cells. Biol. Reprod. 71, 2065-2071. doi: 10.1095/biolreprod.104.028076

Rae, C., and Graham, A. (2008). Fatty acid synthase inhibitor, C75, blocks resistininduced increases in lipid accumulation by human macrophages. Diabetes Obes. Metabol. 10, 1271-1274. doi: 10.1111/j.1463-1326.2008.00962.x

Richa, S., Vikas, Y., Sachin, K., and Neeru, S. (2015). MicroRNA-195 inhibits proliferation, invasion and metastasis in breast cancer cells by targeting FASN, HMGCR, ACACA and CYP27B1. Sci. Rep. 5, 1-15. doi: 10.1038/srep17454

Rivas, R. E. C., Nieto, M. P. C., and Kamiyoshi, M. (2015). Effects of steroid hormone in avian follicles. Asian Australas. J. Anim. Sci. 29, 487-499. doi: 10.5713/ajas. 15.0310

Spicer, L. J., Hamilton, T. D., and Keefer, B. E. (1996). Insulin-like growth factor I enhancement of steroidogenesis by bovine granulosa cells and thecal cells: dependence on de novo cholesterol synthesis. J. Endocrinol. 151, 365-373. doi: 10.1677/joe.0.1510365

Strable, M. S., and Ntambi, J. M. (2010). Genetic control of de novo lipogenesis: role in diet-induced obesity. Crit. Rev. Biochem.Mol. Biol. 45, 199-214. doi: 10.3109/10409231003667500

Sun, L., Yao, Y. Z., Pan, G. F., Zhan, S. H., Shi, W. Q., Lu, T., et al. (2018). Small interfering RNA-mediated knockdown of fatty acid synthase attenuates the proliferation and metastasis of human gastric cancer cells via the mTOR/Gli1 signaling pathway. Oncol. Lett. 16, 594-602. doi: 10.3892/ol.2018. 8648

Vanholder, T., Leroy, J. L., Soom, A. V., Maes, D., Coryn, M., Fiers, T., et al. (2006). Effect of non-esterified fatty acids on bovine theca cell steroidogenesis and proliferation in vitro. Anim. Reprod. Sci. 92, 51-63. doi: 10.1016/j.anireprosci. 2005.05.014

Vanholder, T., Leroy, J. L. M. R., Soom, A. V., Opsomer, G., Maes, D., Coryn, M., et al. (2005). Effect of non-esterified fatty acids on bovine granulosa cell steroidogenesis and proliferation in vitro. Anim. Reprod. Sci. 87, 33-44. doi: 10.1016/j.anireprosci.2004.09.006

Wen, R., Gan, X., Hu, S. Q., Gao, S. Y., Deng, Y., Qiu, J. M., et al. (2019). Evidence for the existence of de novo lipogenesis in goose granulosa cells. Poult. Sci. 98, 1023-1030. doi: 10.3382/ps/pey400

You, B. J., Hour, M. J., Chen, L. Y., Luo, S. C., Hsu, P. H., and Lee, H. Z. (2019). Fenofibrate induces human hepatoma Hep3B cells apoptosis and necroptosis through inhibition of thioesterase domain of fatty acid synthase. Sci. Rep. 9, 1-12. doi: 10.1038/s41598-019-39778-y

Zhang, Y. F., Yuan, Z. Q., Song, D. G., Zhou, X. H., and Wang, Y. Z. (2014). Effects of cannabinoid receptor 1 (brain) on lipid accumulation by transcriptional control of CPT1A and CPT1B. Anim. Gen. 45, 38-47. doi: 10.1111/age. 12078

Conflict of Interest: The authors declare that the research was conducted in the absence of any commercial or financial relationships that could be construed as a potential conflict of interest.

Copyright (c) 2020 Chen, Huang, Hu, Lan, Gan, Gao, Deng, Hu, Li, Hu, He, Liu, Xia and Wang. This is an open-access article distributed under the terms of the Creative Commons Attribution License (CC BY). The use, distribution or reproduction in other forums is permitted, provided the original author(s) and the copyright owner(s) are credited and that the original publication in this journal is cited, in accordance with accepted academic practice. No use, distribution or reproduction is permitted which does not comply with these terms. 